\section{P74 COMPLEMENT VARIATIONS IN SYSTEMIC LUPUS ERYTHEMATOSUS PREGNANCIES}

${ }^{1}$ Francesca Crisafulli, ${ }^{2}$ Laura Andreoli, ${ }^{3}$ Matteo Filippini, ${ }^{2}$ Micaela Fredi, ${ }^{2}$ Maria Chiara Gerardi, ${ }^{3}$ Roberto Gorla, ${ }^{2}$ Maria Grazia Lazzaroni, ${ }^{3}$ Cecilia Nalli, ${ }^{3}$ Marco Taglietti, ${ }^{4}$ Andrea Lojacono, ${ }^{4}$ Sonia Zatti, ${ }^{2}$ Franco Franceschini, ${ }^{2}$ Angela Tincani. ${ }^{1}$ Rheumatology and Clinical Immunology Unit, Spedali Civili of Brescia and University of Pavia, Pavia; ${ }^{2}$ Rheumatology and Clinical Immunology Unit, Spedali Civili, University of Brescia, Brescia; ${ }^{3}$ Rheumatology and Clinical Immunology Unit, Spedali Civili, Brescia; ${ }^{4}$ Obstetrics and Gynecology Unit, Spedali Civili and University of Brescia, Brescia, Italy

\subsection{6/lupus-2020-eurolupus. 119}

Background/Purpose To analyse complement variations during SLE pregnancies, considering pregnancies with Adverse Pregnancy Outcome (APO) and with flares.

Methods Monocentric, retrospective study of 98 SLE patients with 134 pregnancies (including 3 twin pregnancies) prospectively followed by multidisciplinary team (1987-2015). Нypocomplementemia was defined according to the normality range calculated in healthy pregnancies by Reggia et al. ${ }^{1}$

Results APO occurred in $22(16 \%)$ and flares in $11(8 \%)$ pregnancies. The variation of mean $\mathrm{C} 3$ and $\mathrm{C} 4$ levels are shown in table 1 . Notably, both in pregnancies with flares and with APO, there was no increase of C3 between the 2nd and the 3rd trimester and of C4 between the 1st and the 3 rd trimester. In pregnancies with flares the mean levels of C3 and C4 were lower than in pregnancies without flares during the 2 nd and the 3 rd trimester; the mean levels of C4 were also lower in the flare group during pre-conceptional visit. In pregnancies with APO, the variation of C4 levels between the 2nd and the 3rd trimester was lower than in pregnancies without APO $(-3.18$ vs $0.27 ; \mathrm{p}=0.01)$. The frequency of low C4 was higher in pregnancies with flare at pre-conceptional visit, 1 st trimester and $3 \mathrm{rd}$ trimester $(6 / 7$ vs $25 / 103 \mathrm{p}=0.002 ; 8 / 9$ vs $56 / 106 \mathrm{p}=0.04 ; 9 / 11$ vs $33 / 96 \mathrm{p}=0.003)$, as compared with pregnancies without flares.

Conclusions In our cohort, low C4 at pre-conceptional visit seems to predict flares during pregnancies. Lower increase of C4 levels between the $2 \mathrm{nd}$ and the 3 rd trimester could predict an APO.

\section{REFERENCE}

1. Reggia R., Ziglioli T., Andreoli L., et al. Primary anti-phospholipid syndrome: any role for serum complement levels in predicting pregnancy complications?. Rheumatology 2012;51:2186-2190

\section{P75 $\quad$ ANTI-SSA/RO POSITIVITY AND THE RISK OF CONGENITAL HEART BLOCK: OBSTETRIC AND FETAL OUTCOME IN A COHORT OF ANTI-SSA/RO POSITIVE PREGNANT PATIENTS WITH AND WITHOUT AUTOIMMUNE DISEASES}

${ }^{1}$ Micaela Fredi, ${ }^{2}$ Maria Gerosa, $\quad{ }^{1}$ Laura Andreoli, ${ }^{1}$ Tamara Vojanovic, ${ }^{2}$ Cecilia Beatrice Chighizola, ${ }^{3}$ Federica Gazzola, ${ }^{4}$ Andrea Lojacono, ${ }^{4}$ Sonia Zatti, ${ }^{3}$ Laura Trespidi, ${ }^{2}$ Enrico Ferrazzi, ${ }^{2}$ Roberto Caporali, ${ }^{2}$ PierLuigi Meroni, ${ }^{1}$ Franco Franceschini, ${ }^{1}$ Angela Tincani. 'Rheumatology and Clinical Immunology Unit and Clinical and Experimental Science Dept. ASST Spedali Civili and University of Brescia, Brescia; ${ }^{2}$ Istituto Ortopedico Gaetano Pini, University of Milan, Milan; ${ }^{3}$ Dept. per la salute della Donna, Bambino e Neonato, Fondazione Ospedale Maggiore, Milan; ${ }^{4}$ Obstetrics and Gynecology Dept., ASST Spedali Civili and University, Brescia, Italy

\subsection{6/lupus-2020-eurolupus. 120}

Background Neonatal lupus syndrome is an acquired disease caused by the transplacental passage of anti-SSA/Ro antibodies. Congenital heart block (CHB) represent the most serious manifestation. The rate of $\mathrm{CHB}$ in anti-SSA/Ro positive pregnant woman varies among studies ranging from 1 to $5 \%$ in different populations. Aim of our study was to assess the prevalence of $\mathrm{CHB}$ in a cohort of anti-SSA/Ro positive pregnant women prospectively followed up in 2 Italian tertiary referral centers.

Methods Patients underwent monthly clinical examination and data regarding the disease and pregnancy course were recorded. Moreover, fetal heart rates were assessed weekly by Doppler ultrasound from the 14th to the 26th gestational week.

Results Between 2010 and 2018 we recorded data of 286 pregnancies, with the following maternal diagnosis: SLE in 73 (25.3\%), Sjogren Syndrome in $52(18 \%)$, undifferentiated connective tissue disease in $59(20.6 \%)$, asymptomatic Ro/SSA carriers in $50(17.4 \%)$ and other connective tissue disease in

Abstract P74 Table 1 C3 and C4 mean levels (mg/dL) at pre-conceptional visit (T0), 1st trimester (T1), 2nd trimester (T2) and 3rd trimester (T3). Flare during pregnancies were: 2 renal, 4 articular, 6 cutaneous and 1 neurological. APO were defined as: early miscarriage (<10th week), intrauterine fetal death $(>10$ th week), perinatal death ( $<30$ th day of life), pre-eclampsia (PE), severe preterm birth $(<34$ th week)

\begin{tabular}{|c|c|c|c|c|c|c|c|}
\hline & C3 T0 & C3 T1 & C3 T2 & C3 T3 & $p$ TO-T1 & p T1-T2 & p T2-T3 \\
\hline Pregnancies (tot) & 84.5 & 91.0 & 102.4 & 112.4 & $<0.001$ & $<0.001$ & $<0.001$ \\
\hline Pregnancies with flares* & 73.2 & 82.2 & 83.8 & 97.5 & 0.04 & 0.02 & ns \\
\hline Pregnancies without flares* & 85.3 & 91.8 & 104.4 & 114.7 & $<0.001$ & $<0.001$ & $<0.001$ \\
\hline Pregnancies with $\mathrm{APO}^{\S}$ & 84.7 & 91.5 & 98.5 & 106.3 & 0.03 & 0.03 & ns \\
\hline \multirow[t]{2}{*}{ Pregnancies without $\mathrm{APO}^{\S}$} & 84.5 & 90.9 & 102.8 & 112.8 & $<0.001$ & $<0.001$ & $<0.001$ \\
\hline & C4 TO & C4 T1 & C4 T2 & C4 T3 & p T0-T1 & p T1-T2 & p T2-T3 \\
\hline Pregnancies (tot) & 12.9 & 14.5 & 15.8 & 16.0 & $<0.001$ & $<0.001$ & ns \\
\hline Pregnancies with flares** & 8.6 & 11.8 & 10.9 & 11.4 & 0.01 & ns & ns \\
\hline Pregnancies without flares** & 13.2 & 14.7 & 16.3 & 16.6 & $<0.001$ & $<0.001$ & ns \\
\hline Pregnancies with $\mathrm{APO} \mathrm{S}^{\S \S}$ & 13.1 & 16.1 & 15.9 & 14.7 & 0.01 & ns & ns \\
\hline Pregnancies without $A P 0^{\S \S}$ & 12.9 & 14.2 & 15.8 & 16.1 & $<0.001$ & $<0.001$ & ns \\
\hline
\end{tabular}

Comparison of $\mathrm{C} 3$ and $\mathrm{C} 4$ mean levels between pregnancies with fares vs without flares:

${ }^{*}$ T0, T1: p ns. T2, T3: p $0.01{ }^{* *}$ T0: p 0.02. T1: p ns. T2, T3: p 0.01

Comparison of $\mathrm{C} 3$ and $\mathrm{C} 4$ mean levels between pregnancies with APO vs without APO:

§ T0, T1, T2, T3: ns. §§ T0, T1, T2, T3: ns. 
the remaining. In 5 pregnancies $(1.7 \%)$ a third-degree $\mathrm{CHB}$ was diagnosed. In all these 5 pregnancies, anti-SSA/Ro positivity was established after $\mathrm{CHB}$ detection and the mothers were initially labeled as SSA/Ro carriers.

Conclusions Even if the incidence of $\mathrm{CHB}$ as a whole was comparable to that reported in previous studies, none of the pregnancies prospectively followed before and during pregnancy developed CHB. These data suggest that a strict follow up and proper treatment of anti-SSA/Ro positive patients with an established autoimmune disease before and during pregnancy can reduce the risk.

\section{P76 LOW DOSE ASPIRIN TO PREVENT PRE-ECLAMPSIA IN SLE PREGNANCIES - COUNSELLING HELPS TO REALIZE OUR FULL POTENTIAL}

Isabell Haase, Matthias Schneider, Ralph Brinks, Rebecca Fischer-Betz. Policlinic for Rheumatology and Hiller Research Centre for Rheumatology, Heinrich-Heine-University Duesseldorf, Duesseldorf, Germany

\subsection{6/lupus-2020-eurolupus. 121}

Background Women with Systemic Lupus Erythematosus (SLE) face a higher risk of pre-eclampsia, especially those with additional risk factors. Low dose aspirin (LDA) is known to protect against pre-eclampsia in non-autoimmune patients. Consequently, the EULAR recommends starting LDA in those women at risk preconceptionally or latest until gestational week 16. We sought to examine the use of LDA in a realworld cohort in relation to different risk factors and the provision of preconception counselling.

Methods Pregnancies of women with SLE from an outpatient pregnancy clinic were evaluated before and throughout pregnancy. Clinical characteristics including pre-eclampsia risk factors, disease activity (SLEDAI) and medication use were analysed. Association of Aspirin use (latest from week 16 on) with different risk factors or preconception counselling was analysed using $\chi^{2}$ tests.

Results We enrolled 201 pregnancies in 136 women. $57.8 \%$ of pregnancies showed a high-risk profile for pre-eclampsia (history of pre-eclampsia, multifetal gestation, chronic hypertension, lupus nephritis or aPL), another $26.6 \%$ had at least one moderate risk factor (nulliparous, body mass index $>30$ or age $>35$ ). LDA was administered in $43.3 \%$ of pregnancies. LDA use was significantly higher in those with a high-risk profile $(63.5 \%$ vs. 16.7\%) [OR 8.59 (95\%-CI: 4.19-18.62), $\mathrm{p}<0.001$ ], but not in those with a moderate-risk profile. Still, $36.5 \%$ of those at high risk and $83 \%$ of those at moderate risk did not receive

\section{Abstract P76 Table 1}

\begin{tabular}{|c|c|c|c|}
\hline & All pregnancies $(n=201)$ & No LDA therapy $(n=114)$ & LDA therapy $(\mathrm{n}=87)$ \\
\hline \multicolumn{4}{|l|}{ Patient characteristics at conception } \\
\hline Age (years), median (IQR) & $31(28-34)$ & $30.5(27-34)$ & $31(29-33)$ \\
\hline Age $>35, n(\%)$ & $42(20.9 \%)$ & $27(23.7 \%)$ & $15(17.2 \%)$ \\
\hline BMI, median (IQR) & $23(22-24)$ & $23(22-25)$ & $23(22-24)$ \\
\hline $\mathrm{BMI}>30, \mathrm{n}(\%)$ & $12(6.1 \%)$ & $6(5.4 \%)$ & $6(7.0 \%)$ \\
\hline Chronic Hypertension, n (\%) & $34(16.9 \%)$ & $17(14.9 \%)$ & $17(19.5 \%)$ \\
\hline CKD III ${ }^{* 1}$ or higher, $\mathrm{n}(\%)$ & $4(2.2 \%)$ & $2(1.9 \%)$ & $2(2.5 \%)$ \\
\hline Preconception counselling, $n(\%)$ & $131(65.2 \%)$ & $65(57.0 \%)$ & $66(75.9 \%)$ \\
\hline \multicolumn{4}{|l|}{ Year of preconceptional visit } \\
\hline 1995-1999, n (\%) & 23 & $19(82.6 \%)$ & $4(17.4 \%)$ \\
\hline $2000-2004, \mathrm{n}(\%)$ & 49 & $34(69.4 \%)$ & $15(30.6 \%)$ \\
\hline 2005-2009, n (\%) & 49 & $31(63.3 \%)$ & $18(36.7 \%)$ \\
\hline 2010-2014, n (\%) & 58 & $25(43.1 \%)$ & $33(56.9 \%)$ \\
\hline 2015-2019, n (\%) & 22 & $5(22.7 \%)$ & $17(77.3 \%)$ \\
\hline \multicolumn{4}{|l|}{ Obstetrical history and characteristics } \\
\hline Nulliparous, n (\%) & $123(61.2 \%)$ & $60(52.6 \%)$ & $63(72.4 \%)$ \\
\hline Previous fetal loss, n (\%) & $40(19.9 \%)$ & $14(12.3 \%)$ & $26(29.9 \%)$ \\
\hline Previous pre-eclampsia, n (\%) & $16(8 \%)$ & $7(6.1 \%)$ & $9(10.3 \%)$ \\
\hline Multifetal gestation, n (\%) & $8(4.2 \%)$ & $1(0.9 \%)$ & $7(8.5 \%)$ \\
\hline \multicolumn{4}{|l|}{ SLE characteristics } \\
\hline Disease duration (years), median (IQR) & $6.4(2.7-10.8)$ & $6.0(2.2-10.13)$ & $7.0(3.0-11.0)$ \\
\hline SLEDAI, median (IQR) & $2.0(0-4.0)$ & $2.0(0-4.0)$ & $2.0(2.0-4.0)$ \\
\hline Lupus nephritis, n (\%) & $58(28.9 \%)$ & $24(21.1 \%)$ & $34(29.1 \%)$ \\
\hline Anti-dsDNA antibodies, n (\%) & $111(55.5 \%)$ & $57(50.4 \%)$ & $54(62.1 \%)$ \\
\hline Prednisolone therapy, n (\%) & $100(49.8 \%)$ & $57(50.0 \%)$ & $43(49.4 \%)$ \\
\hline Prednisolone (mg/d), median (IQR) & $5.0(5.0-7.25)$ & $5.0(5.0-8.0)$ & $5.0(5.0-5.0)$ \\
\hline \multicolumn{4}{|l|}{ Antiphospholipid status } \\
\hline APS, n (\%) & $31(15.4 \%)$ & $3(2.6 \%)$ & $28(32.2 \%)$ \\
\hline Any positive aPL, n (\%) & $48(24 \%)$ & $7(6.2 \%)$ & $41(47.1 \%)$ \\
\hline LAC, n (\%) & $31(15.5 \%)$ & $3(2.7 \%)$ & $28(32.2 \%)$ \\
\hline $\mathrm{ACL}, \mathrm{n}(\%)$ & $33(16.5 \%)$ & $5(4.4 \%)$ & $28(32.2 \%)$ \\
\hline$\beta 2-G P 1, n(\%)$ & $28(14 \%)$ & $4(3.5 \%)$ & $24(27.6 \%)$ \\
\hline
\end{tabular}

$\mathrm{BMI}=$ body mass index, aPL = Antiphospholipid antibody, LAC = Lupus anticoagulant, $\mathrm{ACL}=$ Anticardiolipin antibody, $\beta 2$-GP1 = $\beta 2$-Glycoprotein I antibody, CKD = chronic kidney disease, IUGR $=$ intrauterine growth restriction; *1 eGFR (MDRD) $<60 \mathrm{ml} / \mathrm{min} / 1.73 \mathrm{~m}^{2},{ }^{\star 2}<36$ weeks of gestation 